\title{
Low expression of PTEN is essential for maintenance of a malignant state in human gastric adenocarcinoma via upregulation of p-AURKA mediated by activation of AURKA
}

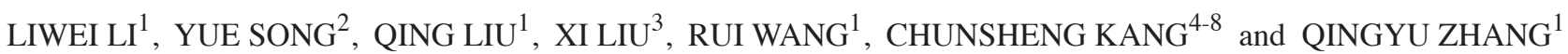 \\ ${ }^{1}$ Department of Gastroenterology, Tianjin Medical University General Hospital; ${ }^{2}$ Department of Gastroenterology, \\ Tianjin Medical University Cancer Institute Hospital; ${ }^{3}$ Department of Gastroenterology, Tianjin Nankai Hospital; \\ ${ }^{4}$ Department of Neurosurgery, Tianjin Medical University General Hospital; ${ }^{5}$ Laboratory of Neuro-Oncology, \\ Tianjin Neurological Institute; ${ }^{6}$ Key Laboratory of Post-Trauma Neuro-Repair and Regeneration in Central Nervous System, \\ Ministry of Education; ${ }^{7}$ Tianjin Key Laboratory of Injuries, Variations and Regeneration of Nervous System, \\ Tianjin 300052; ${ }^{8}$ Chinese Glioma Cooperative Group, Beijing 100050, P.R. China
}

Received October 8, 2017; Accepted February 14, 2018

DOI: $10.3892 / \mathrm{ijmm} .2018 .3544$

\begin{abstract}
Gastric adenocarcinoma remains a life-threatening disease, emphasizing the importance of gaining an improved understanding of signaling pathways involved in this disease, which can lead to the development of novel therapeutic methods targeting common molecular pathways shared across different types of gastric adenocarcinoma. The present study revealed phosphatase and tensin homolog deleted on chromosome 10 (PTEN) and aurora kinase A (AURKA) gene alterations, which were involved in changes in the phenotypes of gastric cancer cells, including increased proliferation by cell counting kit- 8 assay and invasion capacity by Transwell invasion assay, and predicted survival rates by KM Plotter database in gastric cancer. The present study investigated the association between PTEN and AURKA. Western blotting revealed that phosphorylated (p)-AURKA correlated with two target genes, PTEN and AURKA. The downregulation of PTEN by small interfering (si)RNA not only increased the expression of AURKA at the mRNA and protein levels by western blotting and by reverse transcription-quantitative PCR, but also increased the expression of p-AURKA by western blotting and immunofluorescence analysis. In addition, western
\end{abstract}

Correspondence to: Professor Qingyu Zhang, Department of Gastroenterology, Tianjin Medical University General Hospital, 154 Anshan Road, Heping, Tianjin 300052, P.R. China

E-mail: zhangqy@tijmu.edu.cn

Professor Chunsheng Kang, Department of Neurosurgery, Tianjin Medical University General Hospital, 154 Anshan Road, Heping, Tianjin 300052, P.R. China

E-mail: kang97061@gmail.com

Key words: phosphatase and tensin homolog deleted on chromosome 10, aurora kinase A, phosphorylated aurora kinase A, gastric cancer blotting and reverse transcription-quantitative PCR revealed that the downregulation of AURKA affected the expression level of PTEN. Furthermore, PTEN suppressed the malignant phenotypic changes of gastric adenocarcinoma cells by regulating the expression of AURKA inhibited by p-AURKA, suggesting that p-AURKA may be the key mediator of the PTEN-associated activation of AURKA and may be key in maintaining the PTEN-induced malignant state of gastric adenocarcinoma cells. This hypothesis was confirmed by western blotting, and changes were observed in the protein expression of p-AURKA and AURKA under conditions in which cells were treated with either MLN8237 or si-PTEN transfection only, or with si-PTEN transfection and MLN8237. Knockdown of the expression of PTEN altered the expression of p-AKT, p-glycogen synthase kinase $3 \beta$ and $\beta$-catenin, which are genes that have been reported to be involved in the development of gastric adenocarcinoma. The present study confirmed that p-AURKA is important in the development of gastric adenocarcinoma and revealed a novel functional link between PTEN, AURKA and p-AURKA activation. The results also suggest a novel drug design strategy in targeting PTEN and AURKA for more specific gastric cancer cell death that spares normal cells.

\section{Introduction}

Globally and in high socio-demographic index (SDI) countries, stomach cancer ranked fifth highest for cancer incidence and third highest for cancer-associated mortality in 2015. In high-middle, middle, low-middle, and low SDI countries, stomach cancer incidence ranked third. For cancer-associated mortality in high-middle, middle, and low SDI countries, stomach cancer ranked as the third highest cause. For low-middle SDI countries, stomach cancer was the second highest cause of cancer-associated mortality (1). According to the National Cancer Institute's Surveillance, Epidemiology, and End Results program, 26,370 new cases of gastric cancer were expected to be diagnosed in 2016, with an estimated 
mortality rate of 10,730 and a low survival rate of $30.4 \%$ at 5 years (2), indicating the need for novel therapeutic approaches and an understanding of the biological mechanisms of stomach cancer. In previous years, the treatment of stomach cancer has focused on its surgical resection. Surgical removal remains the optimal treatment in patients with resectable tumors, and the overall survival (OS) range is between 5 and $90 \%$ depending on disease staging at diagnosis (3). However, the recurrence rate of gastric cancer is $\sim 20-50 \%$ in all gastric resections (4). Improved prognosis has been achieved through the use of adjuvant chemoradiotherapy and perioperative chemotherapy. However, the majority of cases of gastric cancer are diagnosed in an advanced and unresectable stage; therefore, the optimal treatment for these patients includes chemotherapy, novel target therapies and supportive care (5). Although there are a large number of potential therapeutic targets, only a limited number are currently known. Therefore, the development of more specifically targeted and less toxic therapeutic methods, particularly those that target common molecular pathways associated with disease progression and maintenance, and those shared across a wide range of gastric cancer types, is crucial for patients with gastric cancer.

Aurora kinases (AURKs) are a family of conserved serine/threonine protein kinases, which are involved in several stages of mitosis (6); three of family members are AURKA, AURKB and AURKC. These members are expressed in mitotic and meiotic cells (7), and AURKA localizes to the duplicate centrosomes at the beginning of the $\mathrm{S}$ phase, shifts to the bipolar spindle microtubules during mitosis, and moves to perinuclear components of the daughter cell at the end of mitosis (8). AURKA is also important in tumorigenesis and tumor progression. The overexpression of AURKA has been found in several malignancies, including breast cancer, esophageal squamous cell carcinoma and bladder cancer (9-11), According to a previous study, the overexpression of AURKA was significantly increased in differentiated gastric carcinoma, suggesting that the high expression of AURKA is involved in the development and progression of differentiated gastric carcinoma (12).

Phosphatase and tensin homolog deleted on chromosome 10 (PTEN) is a tumor suppressor gene, which functions primarily as a cellular lipid phosphatase (13). According to previous studies, the overexpression of PTEN is protective against cancer (14). PTEN can dephosphorylate phosphatidylinositol 3,4,5-trisphosphate, transforming it into phosphatidylinositol-4,5-bisphosphate (15), and directly antagonizes the phosphoinositide 3-kinase (PI3K)/Akt signaling pathway, which is involved in cell survival, cell proliferation, angiogenesis and anabolic metabolism (16). However, PTEN has been found to be frequently mutated in various types of human cancer (17-19). Studies have shown that alterations of PTEN by inactivating mutations and/or chromosomal deletions contribute to the progression of gastric cancer (20).

The present study showed for the first time, to the best of our knowledge, that PTEN downregulated the expression of phosphorylated (p)-AURKA and further affected the activation of AURKA. In addition, the knockdown of the expression AURKA led to decreased expression of p-AURKA, which further increased the expression of PTEN, similar to the results using the AURKA inhibitor alisertib (MLN8237).
Additionally, the aberrant expression of PTEN and AURKA induced malignant changes in the phenotype of gastric cancer cells. The downregulation of PTEN and use of AURKA inhibitor MLN8237 decreased the expression of p-AURKA in MGC-803 and SGC-7901 cells. Knockdown of the expression of PTEN led to significant changes in the expression levels of several other important genes of the PI3K/AKT/glycogen synthase kinase $3 \beta(\mathrm{GSK} 3 \beta) / \beta$-catenin signaling pathway, which are associated with the development of gastric cancer, including p-AKT, p-GSK3 $\beta$ and $\beta$-catenin. The present study showed that p-AURKA is crucial in gastric cancer and is the first to discuss how p-AURKA acts as a key molecule in the activation of PTEN-associated regulation of AURKA.

\section{Materials and methods}

Cell lines and culture. The MGC803 and SGC7901 human gastric cancer cell lines were purchased from China Academia Sinica Cell Repository (Shanghai, China) and cultured in RPMI-1640 medium supplemented with $10 \%$ fetal bovine serum (Gibco; Thermo Fisher Scientific, Inc., Waltham, MA, USA). The GES-I cells were purchased from China Academia Sinica Cell Repository (Shanghai, China) and cultured in Dulbecco's modified Eagle's medium (DMEM) supplemented with $10 \%$ fetal bovine serum (Gibco; Thermo Fisher Scientific, Inc.). The cells were cultivated under in $5 \% \mathrm{CO}_{2}$ at $37^{\circ} \mathrm{C}$.

Antibodies and other reagents. Primary antibodies mouse anti-AURKA (cat. no. ab13824; 1:1,000), rabbit anti-AKT1 (cat.no. ab32505; 1:5,000), rabbit anti-p-AKT1 (s473; cat. no. ab81283; 1:5,000), rabbit anti-GSK3 $\beta$ (cat. no. ab93926; 1:2,000), rabbit anti-p-GSK3 $\beta$ (s9; cat. no. ab75814; 1:10,000) and rabbit anti- $\beta$-catenin (cat. no. ab32572; $1: 5,000$ ) were purchased from Abcam (Cambridge, UK). Primary antibodies rabbit anti-p-AURKA (Thr288; cat. no. 3079; 1:1,000), rabbit anti-PTEN (cat. no. 9188; 1:1,000) and rabbit anti-p- $\beta$-catenin (Ser675; cat. no. 4176; 1:1,000) were purchased from Cell Signaling Technology, Inc. (Danvers, MA, USA). Mouse anti-GAPDH (cat. no. TA309157; 1:1,000) antibody was purchased from Zhongshan Golden Bridge Biotechnology (Beijing, China). Secondary antibodies included anti-rabbit (cat. no. ZB-2301; 1:5,000) and anti-mouse (cat. no. ZB-2305; 1:5,000) purchased from Zhongshan Golden Bridge Biotechnology (Beijing, China). The AURK inhibitor alisertib was purchased from Selleck Chemicals (Houston, TX, USA).

Immunocytochemistry. All tissue samples were gathered from the isolated specimens of surgical patients of the Tianjin Medical University General Hospital (Tianjin, China) between December 2016 and May 2017, and the tissue type includes the normal gastric mucosa and the gastric carcinoma tissues. These patients are diagnosed with gastric adenocarcinoma. The tumor tissue is from the gastric antrum, cardia and gastric corpus. Formalin-fixed tissue samples were prepared as paraffin-embedded sections, and immunostaining was performed on the sections using the avidin-biotin-complex method. The tissue sections were incubated with the indicated primary antibodies (anti-AURKA antibody, anti-PTEN antibody, anti-AKT antibody, anti-p-AKT antibody, anti-GSK3 $\beta$ antibody, anti-p-GSK3$\beta$ antibody, and anti- $\beta$-catenin 
antibody) overnight at $4^{\circ} \mathrm{C}$. The tissues were incubated with secondary antibodies at a dilution of $1: 100$ for $1 \mathrm{~h}$ at $37^{\circ} \mathrm{C}$, washed with PBS, incubated with the avidin-biotin complex (ABC)-peroxidase for $1 \mathrm{~h}$, and washed with PBS again. The tissues were counterstained with diaminobenzidine buffer, hematoxylin and $0.1 \%$ TRIS and were then dehydrated in alcohol, and visualized under an Olympus light microscope (magnification, x200) (Olympus Corporation, Tokyo, Japan).

Immunofluorescence analysis. Cells $\left(3 \times 10^{4} / 500 \mu \mathrm{l} /\right.$ well) transfected with si-AURKA and si-PTEN were seeded in 24-well plates chamber for $24 \mathrm{~h}$ at $37^{\circ} \mathrm{C}$, washed three times with PBS and fixed with $4 \%$ paraformaldehyde in PBS. The cells were then washed three times with PBS, followed by blocking with $5 \%$ bovine serum albumin (BSA; Beijing Solarbio Science \& Technology Co., Ltd., Beijing, China) for $30 \mathrm{~min}$. The cells were incubated with specific primary antibodies against p-AURKA $(1: 1,600)$ overnight at $4^{\circ} \mathrm{C}$, following which the cells were washed three times with PBS and hybridized with fluorescently conjugated secondary antibody (TRITC; 1:200) for $2 \mathrm{~h}$ at room temperature. The nuclei were stained with DAPI for $15 \mathrm{~min}$ at room temperature and visualized under an Olympus fluorescence microscope (magnification, x400). (Olympus Corporation).

Transwell invasion assay. The Matrigel matrix was diluted with serum-free (DMEM (Gibco; Thermo Fisher Scientific, Inc.) and layered into the upper well of a Transwell chamber. Following incubation for $30 \mathrm{~min}$ at $37^{\circ} \mathrm{C}$, the Matrigel solidified and served as an extracellular matrix for tumor cell invasion analysis. Cells $\left(5 \times 10^{4} / 500 \mu \mathrm{l} /\right.$ well $)$ were seeded in the upper chamber of the 24-well Transwell plate and incubated for $48 \mathrm{~h}$ at $37^{\circ} \mathrm{C}$ in $5 \% \mathrm{CO}_{2}$. The cells were fixed with paraformaldehyde for $15 \mathrm{~min}$ and stained with crystal violet for $10 \mathrm{sec}$. The chambers were detected under an Olympus microscope at 200x magnification, using three randomly selected fields to count the number of cells.

Cell proliferation measurement using a CCK-8 assay. The tumor cells were transfected with si-AURKA and si-PTEN, and seeded into 96-well plates (2,000 cells/well), with three wells included for each group. The cells were incubated for 4 days at $37^{\circ} \mathrm{C}$ in an environment with $5 \% \mathrm{CO}_{2}$. Subsequently, $10 \mu \mathrm{l}$ of CCK-8 solution was added to each well, followed by additional incubation for $1 \mathrm{~h}$. The absorbance values were measured on a spectrophotometer at a wavelength of $450 \mathrm{~nm}$.

Transient transfection. The sequences of si-AURKA and si-PTEN were designed by Shanghai GenePharma Co., Ltd. (Shanghai, China). The targeted sequences were as follows: Negative control, forward 5'-UUCUCCGAACGUGUCAGU TT-3' and reverse 5'-ACGUGACACGUUCGGAGAATT-3'; si-AURKA, forward 5'-GAAGAGAGUUAUUCAUAGAdt dt-3' and reverse 5'-TdTCUUCUCUCAAUAAGUAUCU-3'; and si-PTEN, forward 5'-GCGUAUACAGGAACAAUATT-3' and reverse 5'-UAUUGUUCCUGUAUACGCCTT-3'. Prior to transfection, cells $\left(25 \times 10^{4} / 2 \mathrm{ml} /\right.$ well $)$ were cultured for $24 \mathrm{~h}$ at $37^{\circ} \mathrm{C}$ in an environment containing $5 \% \mathrm{CO}_{2} . \mathrm{X}$-tremeGENE siRNA transfection reagent $(5 \mu \mathrm{l})$ was added with a combination of $50 \mu \mathrm{l}$ serum-free DMEM and $5 \mu \mathrm{l}$ siRNA. Transfection of the MGC803 and SGC7901 cells was performed using the above prepared reagent in 6-well plates. Following incubation for $48 \mathrm{~h}$, the cells were used in subsequent experiments.

Reverse transcription-quantitative polymerase chain reaction $(R T-q P C R)$ analysis. Total RNA was extracted from the prepared cells using TRIzol reagent. The RNA $(1 \mu \mathrm{g})$ was then reverse transcribed into cDNA using a Promega Reverse Transcription kit (Promega Corporation, Madison, WI, USA). The RT-PCR sample contained 1 ng cDNA,10 $\mu$ SYBR-Green PCR Master Mix (Applied Biosystems; Thermo Fisher Scientific, Inc.) and $2 \mu \mathrm{M}$ primers, and was analyzed using the ABI StepOne Real-Time PCR system (Applied Biosystems; Thermo Fisher Scientific, Inc.). The PCR conditions were as follows: 40 cycles of $95^{\circ} \mathrm{C}$ for $10 \mathrm{~min}, 95^{\circ} \mathrm{C}$ for $15 \mathrm{sec}, 55^{\circ} \mathrm{C}$ for $40 \mathrm{sec}$. The primer sequences used were as follows: GAPDH, forward, 5'-GGAGCCAGATCCCTCCAAAAT-3' and reverse, 5'-GGCTGT TGTCATACT TCTCATGG-3'; PTEN-001, forward, 5'-AAAGACTTGAAGGCGTATACAGGAA-3' and reverse, 5'-ATGTCTTTCAGCACAAAGATTGTA-3'; PTEN-002, forward, 5'-ACATTATTGCTATGGGATTTC CTGC-3' and reverse, 5'-ATGTCTTTCAGCCAAAGATTG TA-3'; PTEN-003, forward, 5'-TGAAGGCGTATACAGGAA CAATA-3' and reverse, 5'-ATGTCTTTCAGCACAAAG ATTGTAT-3'; AURKA-001, forward, 5'-TCCCACCTTCGG CATCCTA-3' and reverse, 5'-CGAATGACAGTAAGACAG GGC-3', AURKA-002, forward, 5'-TACAGTCCCACCTTC GGCAT-3' and reverse, 5'-CAGGGCATTTGCCAATTC TG-3'; and AURKA-003, forward, 5'-TACAGTCCCACC TTCGGCA-3' and reverse, 5'-GACAGGGCATTTGCCAAT TCTG-3'. The results of PCR were analyzed using the $2^{-\Delta \Delta C T}$ method (21).

Western blot analysis. Following incubation for $48 \mathrm{~h}$ post-transfection, the MGC803 and SGC7901 cells were trypsinized and washed three times with cold PBS, and were then lysed on ice for $30 \mathrm{~min}$ with RIPA buffer. The lysate was collected and centrifuged at $12,000 \mathrm{x}$ g at $4^{\circ} \mathrm{C}$ for $15 \mathrm{~min}$. The protein concentrations were measured using a BCA protein assay kit. A total of $20 \mathrm{mg}$ proteins were separated using $10 \%$ SDS-PAGE, followed by transfer onto polyvinylidene difluoride membranes (EMD Millipore, Billerica, MA, USA). The membranes were blocked for $1 \mathrm{~h}$ in BSA, and then incubated with the appropriate antibodies at $4^{\circ} \mathrm{C}$ overnight. The primary antibodies against PTEN, AURKA, p-AURKA, AKT1, p-AKT1, GSK3 $\beta$, p-GSK3 $\beta$ and $\beta$-catenin were diluted with BSA to a suitable concentration $(1: 1,000)$. Secondary antibodies were added for incubation for $1 \mathrm{~h}$ at room temperature (1:4,000 dilution).

Acquisition and analysis of the database. The clinical features and survival data of patients with pancreatic cancer were obtained from The Cancer Genome Atlas (TCGA) database (http://cancergenome.nih.gov/). The cBioPortal for Cancer Genomics provided visualization, analysis, and the ability to download large-scale cancer genomics data sets (http://cbioportal.org). The data necessary to analyze PTEN and AURKA gene alterations, gene co-expression, and gene enrichment in the cBioPortal for Cancer Genomics was obtained from TCGA. To evaluate the association between the presence of 
different genes and patient clinical outcome, the KM Plotter online tool (http://www.kmplot.com) was used for different gastric cancer subtypes.

Statistical analysis. GraphPad Prism 7.0 software (GraphPad Software, Inc., La Jolla, CA, USA) was used for statistical analysis. Data in each experimental group are presented as the mean \pm standard deviation and the difference between groups was analyzed by one-way analysis of variance or a two-tailed Student's t-test. Kaplan-Meier analysis was applied to analyze the effect of PTEN and AURKA on the survival rates of patients with gastric cancer. $\mathrm{P}<0.05$ was considered to indicate a statistically significant difference.

\section{Results}

PTEN and AURKA gene alterations in 478 studies using the cBioPortal online resource. The data of 478 patients were collected from TCGA, and the PTEN and AURKA gene alterations were analyzed in the cBioPortal for Cancer Genomics. As shown in Fig. 1, PTEN alterations, including amplifications, deep deletions, truncating mutations and missense mutations, were observed in 50 patients and accounted for $10 \%$ of the total mutations in all patients. In addition, two alterations (amplification and missense mutations) of AURKA were detected and visualized in 33 patients, accounting for $7 \%$ of the total mutations in all patients (Fig. 1A and B). AURKA and PTEN gene alterations have been found in mucinous stomach adenocarcinoma, tubular adenocarcinoma of the stomach, signet ring cell carcinoma of the stomach, stomach adenocarcinoma and diffuse-type stomach adenocarcinoma. PTEN deletions accounted for the majority of alterations, with the highest percentage of mutated cases within one cancer type being $25 \%$ and the percentage of mutated cases for all pathologies being $>38 \%$. However, there were few PTEN amplifications in the 478 samples on examination using cBioPortal, accounting for $1.4 \%$ of the mutated cases (Fig. 1C). AURKA amplifications accounted for the majority of alterations in mucinous stomach adenocarcinoma, with $18.2 \%$ of tumors carrying this alteration (TCGA, provisional data). Furthermore, AURKA amplification was common in the three specific pathological types of stomach adenocarcinoma (Fig. 1D).

PTEN and AURKA are aberrantly expressed in gastric cancer cells. AURKA has been reported to be involved in the formation of several types of gastrointestinal cancer (22-25). However, the mechanism of interaction between AURKA and PTEN in gastric cancer has not been addressed. To investigate this, the present study first examined expression levels of PTEN and AURKA through RT-qPCR, western blot and immunocytochemical analyses in gastric carcinoma and normal mucosa samples. The immunocytochemistry showed that the expression of AURKA in gastric carcinoma was higher, compared with that in normal gastric mucosa, whereas the expression of PTEN in the normal gastric mucosa was higher, compared with that in gastric carcinoma (Fig. 2A). Furthermore, The immunocytochemistry reveals that the expression levels of $\mathrm{p}$-AKT, $\mathrm{p}$-GSK3$\beta$, AKT and $\beta$-catenin in normal gastric mucosa and gastric carcinoma also differed (Fig. 2A). In addition, it was found that the expression of
AURKA was elevated in the MGC803 and SGC7901 cells at the mRNA and protein levels, compared with the normal GES-I cells $(\mathrm{P}<0.05$; Fig. 2B-D), indicating that AURKA may be a key molecule in the pathogenesis of gastric cancer, as in other malignant tumors.

Studies have shown that PTEN and AURKA share certain common signaling pathways in malignant tumors (26-31). Therefore, the present study analyzed the expression of PTEN in MGC803 and SGC7901 cells. A decreased mRNA level of PTEN was observed, and the opposite mRNA expression pattern was observed for AURKA, as determined by RT-qPCR analysis $(\mathrm{P}<0.05$; Fig. $2 \mathrm{~B}$ and $\mathrm{C})$. The protein levels of PTEN were also decreased in the MGC803 and SGC7901 cells (Fig. 2D), suggesting a potential functional association between PTEN and AURKA. Therefore, MGC803 and SGC7901 cells were used in subsequent loss- and gain-of-function experiments to further investigate the correlation between PTEN and AURKA.

PTEN and AURKA maintain malignant phenotypes and predict survival rate in gastric cancer. To examine the effect of PTEN and AURKA on the malignant phenotype of gastric cancer cells, MGC803 and SGC7901 cells were transfected with PTEN siRNA or AURKA siRNA, and the cells were monitored for malignant phenotype changes. As shown in Fig. 3A and B, compared with the control vector-transfected cells, transient transfection with si-PTEN led to a marked increase in proliferation in the two gastric cancer cell lines, whereas the knockdown of AURKA resulted in decreased proliferation, compared with that in the untreated group. In addition, the Transwell invasion assays showed that the reduction in the expression of PTEN following transfection with si-PTEN led to an increase in the invasion capacity of MGC803 and SGC7901 cells, whereas the knockdown of AURKA led to significant changes in the invasion capacity of the two cell types (Fig. 3C).

Although the overall incidence of gastric cancer has been decreasing, it remains one of the most common types of malignancy and is the leading cause of cancer-associated mortality worldwide. To investigate whether the gene expression of PTEN and AURKA can predict survival rates of patients with gastric cancer, the present study analyzed the gene expression of PTEN and AURKA in patients with gastric cancer using the KM Plotter. The KM Plotter is a public database containing information from 1,065 patients with gastric cancer to permit the investigation of gene associations with OS. The samples were segregated into high and low expression groups. It was observed that patients with a high expression of PTEN were predicted to have improved OS (log-rank $\mathrm{P}=2.5 \mathrm{e}-09)$. The median survival time of the PTEN high-expressing group was 123.6 months, whereas that of the low expression group was 24.9 months (Fig. 3D). However, patients with gastric cancer with a high expression of AURKA had poor OS, compared with patients with a low expression of AURKA (log-rank $\mathrm{P}=0.0028$ ). The median survival time of the AURKA high-expressing group was 29.5 months, and that of the low-expressing group was 27.4 months (Fig. 3E). These results revealed that PTEN and AURKA are crucial in the maintenance of malignant phenotypes in gastric cancer cells. The expression levels of PTEN and AURKA can determine 


\section{A PTEN

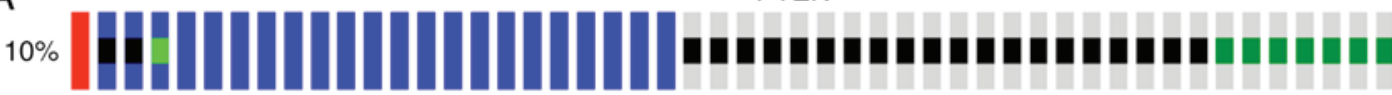 Amplification Deep deletion $\mathbf{a}$ Truncating mutation (putative driver) $\square$ Missense mutation (putative driver) Missense mutation (putative driver)}

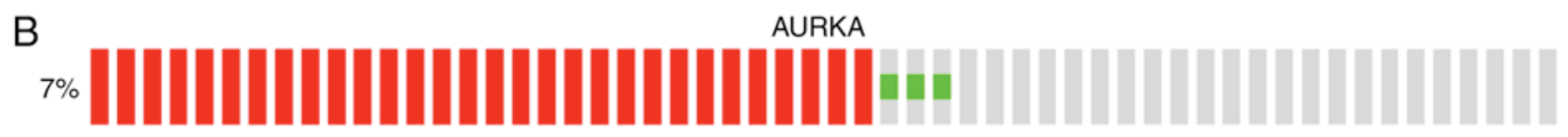

Amplification $\square$ Missense mutation (putative driver)

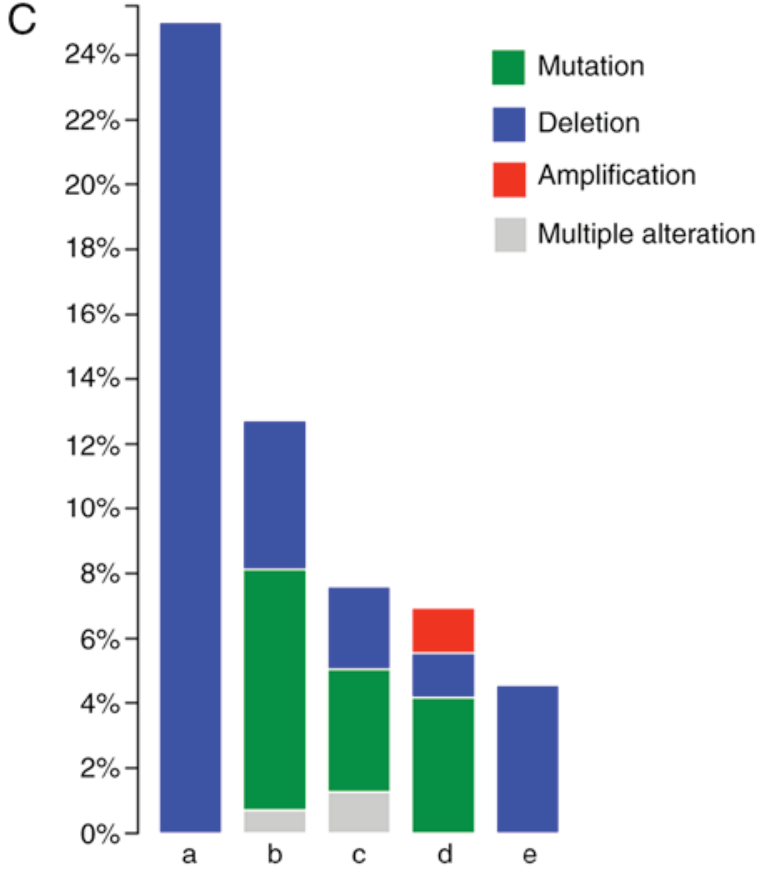

a. Papillary stomach adenocarcinoma

b. Stomach adenocarcinoma

c. Tubular stomach adenocarcinoma

d. Diffuse type stomach adenocarcinoma

e. Mucinous stomach adenocarcinoma

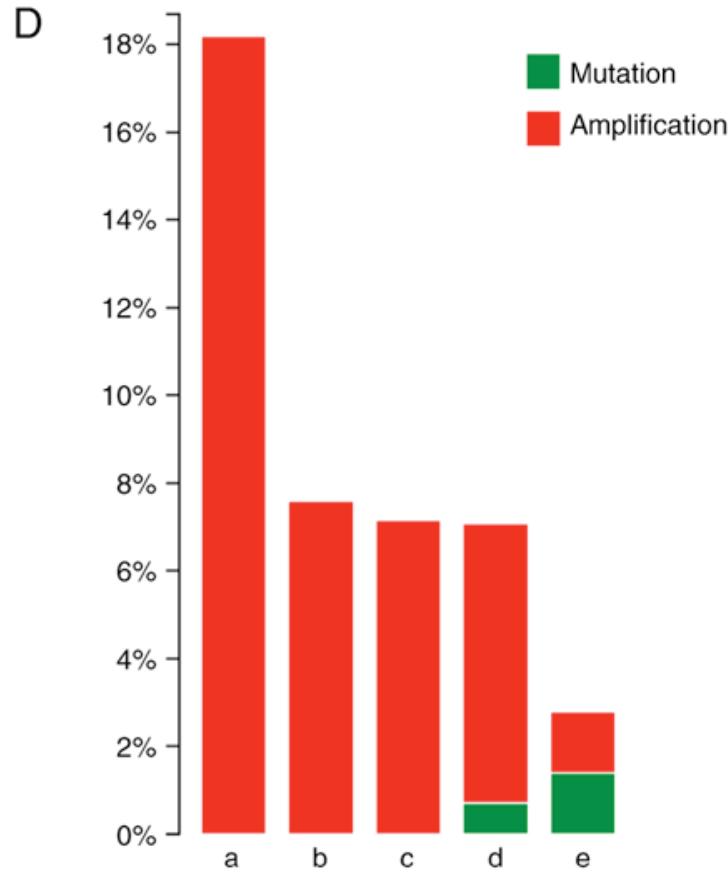

a. Mucinous stomach adenocarcinoma

b. Tubular stomach adenocarcinoma

c. Signet ring cell carcinoma of the stomach

d. Stomach adenocarcinoma

e. Diffuse type stomach adenocarcinoma

Figure 1. Analysis of PTEN and AURKA gene alterations by cBioPortal Cancer Genomics. Genetic alterations in (A) PTEN and (B) AURKA. Red represents amplification, blue represents a deep deletion, black represents a truncating mutation, dark green represents a missense mutation, and light green represents missense mutation. Genomic alteration frequency of (C) PTEN and (D) AURKA in 478 cancer patients. Dark green represents a mutation, blue represents a deletion, red represents an amplification, and gray represents multiple alterations. Data were obtained from the cBioPortal for Cancer Genomics. PTEN, phosphatase and tensin homolog deleted on chromosome 10; AURKA, aurora kinase A.

the clinical progression and outcome of patients with gastric cancer.

Functional link between AURKA and PTEN in gastric cancer. In the present study, data were collected from TCGA to confirm the connection between PTEN and AURKA by using cBioPortal. Pearson's correlation analysis was performed to investigate the correlations between the expression of PTEN and AURKA. The results revealed that the expression of PTEN was significantly negatively correlated with the expression of
AURKA (Fig. 4A). It was also observed that the mRNA expression of PTEN was enriched in the AURKA-unaltered group, and that the mRNA expression of AURKA was enriched in the PTEN-altered group (Fig. 4B).

The outcomes of the above experiments on the expression of PTEN and AURKA in gastric cancer cell lines also suggested a close association between the overexpression of PTEN and AURKA in gastric cancer cells. To further examine the interaction mechanism of PTEN and AURKA, the present study analyzed the gene and protein expression 
A

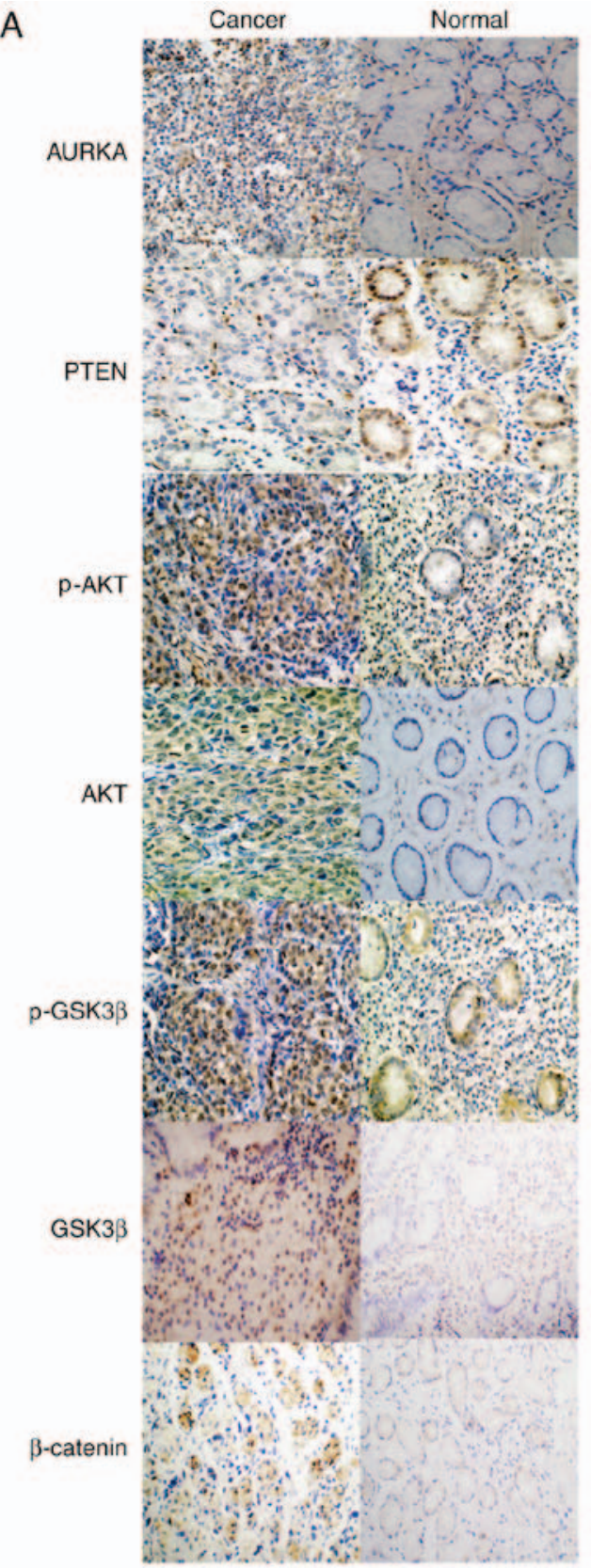

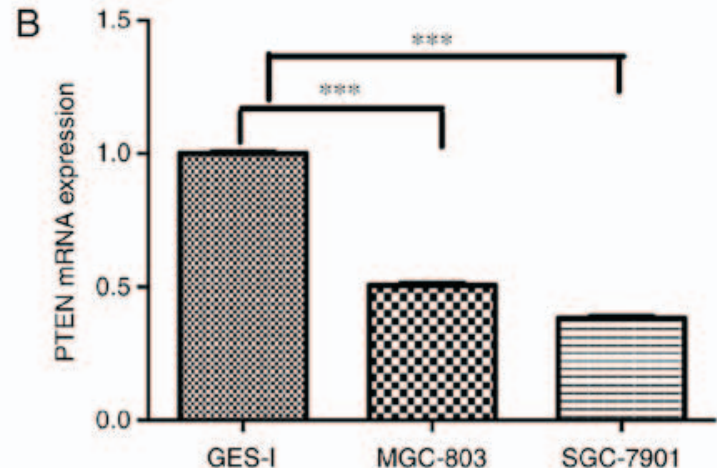

C
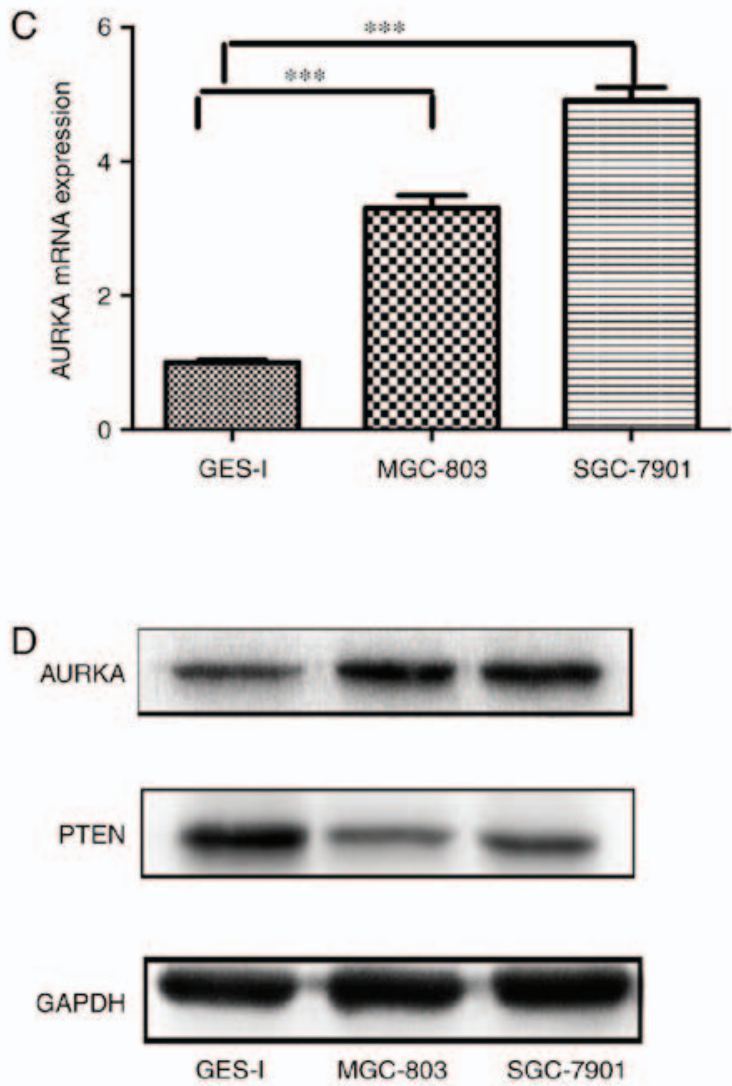

Figure 2. PTEN and AURKA are overexpressed in gastric cancer cell lines. (A) Immunocytochemical analysis of the expression of PTEN, AURKA, p-AKT, AKT, p-GSK3 $\beta$, GSK3 $\beta$ and $\beta$-catenin in patients with gastric cancer (magnification, x200). Analysis of expression levels of (B) PTEN and (C) AURKA in MGC803 and SGC7901 cells, determined using reverse transcription-quantitative polymerase chain reaction analysis $\left({ }^{* * *} \mathrm{P}<0.001\right)$. (D) Western blot analysis of protein expression of PTEN and AURKA in MGC803 and SGC7901 cells. PTEN, phosphatase and tensin homolog deleted on chromosome 10; AURKA, aurora kinase A; GSK3 $\beta$, glycogen synthase kinase $3 \beta$; p-, phosphorylated.

levels of PTEN and AURKA in MGC803 and SGC7901 cells following transfection by si-PTEN and si-AURKA. As shown in Fig. 4C and D, the results showed that the expression of AURKA was markedly higher in the si-PTEN-treated cells than that in control cells at the mRNA level in the gastric cancer cell lines $(\mathrm{P}<0.0001)$. By contrast, compared with the control vector-transfected gastric cancer cells, the expression of PTEN in the si-AURKA-transfected cells was increased $(\mathrm{P}<0.0001)$. In addition, transient transfection with si-AURKA led to an increase in the expression of PTEN at the protein level. It was also found that a reduction in PTEN resulted in the overexpression of AURKA in MGC803 and SGC7901 cells (Fig. 4E and F). These results supported the hypothesis that
PTEN may regulate the expression or function of AURKA, and that changes in the expression or function of AURKA, in turn, may affect the activity of PTEN.

PTEN mediates the AURKA-associated maintenance of a malignant state by activating $p$-AURKA. Previous studies have shown that the autophosphorylation of AURKA is a key regulatory mechanism in centrosomes in the early stages of mitosis, and that the autophosphorylation of p-AURKA creates an activation loop, which increases the catalytic activity of AURKA (32). The present study hypothesized that PTEN can inhibit the activation of AURKA by suppressing the formation of p-AURKA. Therefore, a potential regulatory 

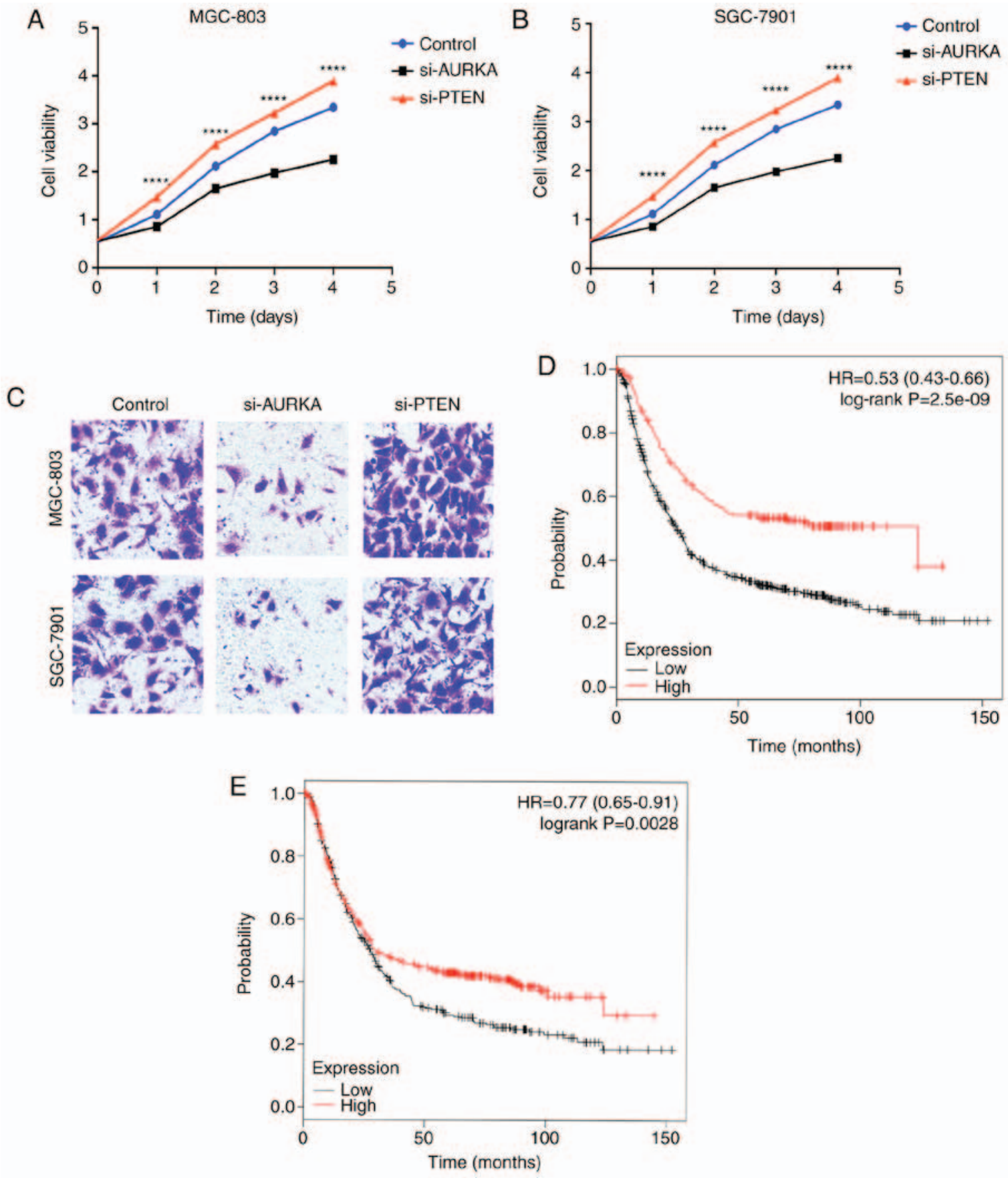

Figure 3. PTEN and AURKA maintain a malignant state in gastric cancer cells and predict survival in patients with gastric cancer. Cell proliferation in si-AURKA- and si-PTEN-transfected (A) MGC803 and (B) SGC7901 cells. The gastric cancer cell growth curves were determined using a CCK-8 assay. (C) Transwell assays for the invasion of si-AURKA- and si-PTEN-transfected MGC803 and SGC7901 cells. The reduction in the expression of PTEN led to increased invasion capacity of gastric cancer cells, whereas AURKA knockdown resulted in decrease invasion capacity of gastric cancer cells (magnification, x200). Comparison of overall survival in (D) PTEN and (E) AURKA high-expressing and low-expressing groups of gastric cancer using the KM Plotter online tool. ${ }^{* * * *} \mathrm{P}<0.0001$ vs. control. PTEN, phosphatase and tensin homolog deleted on chromosome 10; AURKA, aurora kinase A; si-, small interfering RNA.

association among these three proteins was investigated. First, the protein expression of p-AURKA was investigated in conditions of siRNA-induced knockdown of PTEN and knockdown of AURKA in MGC803 and SGC7901 cells. The knockdown of PTEN caused a marked elevation in expression levels of p-AURKA and AURKA in the gastric cancer cell lines. When AURKA was inhibited by transient siRNA transfection in the MGC803 and SGC7901 cells, the protein level of p-AURKA decreased (Fig. 4E and F). The immunofluorescence analysis suggested that si-PTEN treatment in the MGC803 and
SGC7901 cell lines resulted in the increased expression of p-AURKA in the nucleus, whereas the expression level of p-AURKA in the nucleus decreased when the gastric cancer cells were treated with si-AURKA (Fig. 5A). Based on these observations, it was hypothesized that p-AURKA is a downstream target of PTEN. Of note, these outcomes also demonstrated a novel role of p-AURKA in mediating the PTEN-induced activation of AURKA.

To confirm the above hypothesis, a double variable experiment was performed to analyze the effect of PTEN on the 

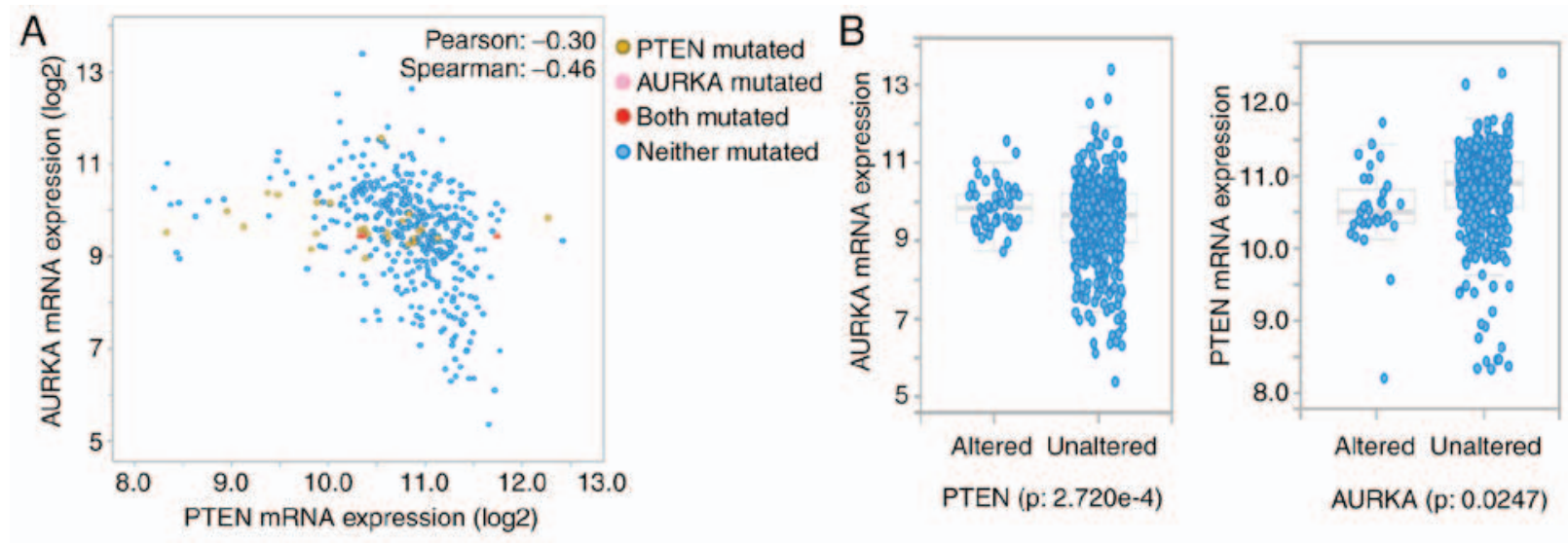

Altered Unaltered
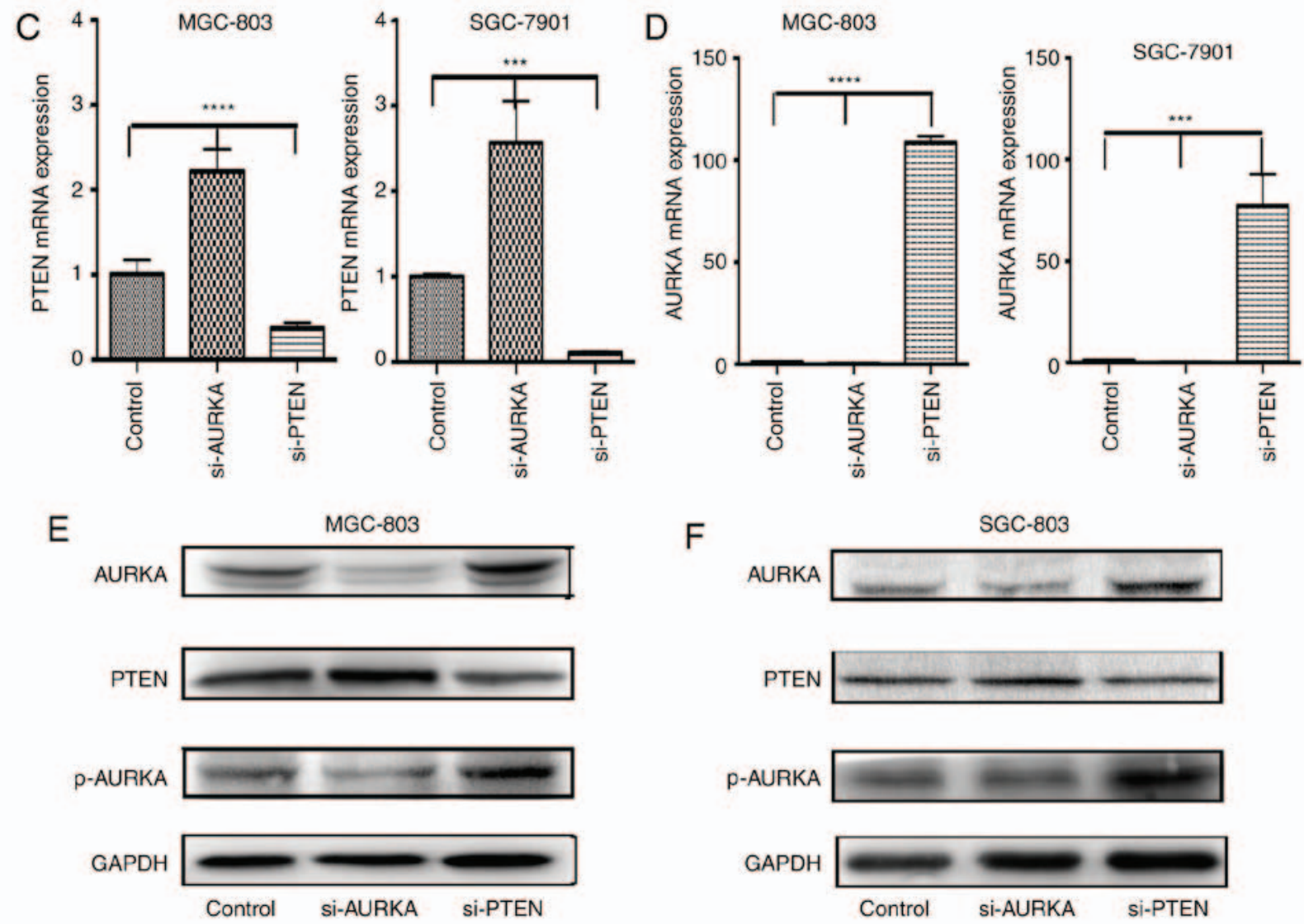

Figure 4. Functional association between AURKA and PTEN in gastric cancer. (A) Correlation between PTEN and AURKA. Pearson's correlation analysis revealed that the expression of AURKA was significantly correlated with the expression of PTEN ( $r=-0.30, P=0.05$; from the cBioPortal for Cancer Genomics). (B) Enrichment in mRNA expression levels of PTEN and AURKA (P<0.05; from the cBioPortal for Cancer Genomics). mRNA expression levels of (C) PTEN and (D) AURKA in MGC803 and SGC7901 cells transfected with si-AURKA and si-PTEN (P<0.05). Analysis of the expression of PTEN, AURKA and p-AURKA by western blot analysis in (E) MGC803 and (F) SGC7901 cells transfected with si-AURKA and si-PTEN. ${ }^{* * *}$ P $<0.001$ and ${ }^{* * * *}$ P $<0.0001$ vs. control, si-AURKA and si-PTEN. PTEN, phosphatase and tensin homolog deleted on chromosome 10; AURKA, aurora kinase A; si-, small interfering RNA; p-, phosphorylated.

expression of p-AURKA and thus the activity of AURKA. Alisertib (MLN8237) is known to selectively inhibit AURKA, markedly inhibit the phosphorylation of AURKA, and lead to a reduction in the level of p-AURKA; however, in the present study, there was a significant increase in the level of AURKA when treated with ALS (33). As shown in Fig. 5B and C, the MGC803 and SGC7901 cells were treated with either MLN8237 or si-PTEN, or with si-PTEN and MLN8237 $(10 \mu \mathrm{M})$, and the protein expression levels of p-AURKA and AURKA were examined in these different conditions. It was observed that the expression of AURKA increased in cells treated with MLN8237 and with si-PTEN transfection plus MLN8237 $(10 \mu \mathrm{M})$ treatment. The expression of AURKA increased more markedly in cells transfected with si-PTEN plus MLN8237 $(10 \mu \mathrm{M})$ treatment, compared with the other groups. However, under conditions of si-PTEN transfection plus MLN8237 treatment, the opposite effect was observed on the protein levels of p-AURKA (Fig. 5B and C) in the MGC803 and SGC7901 cells. In addition, the expression of p-AURKA was increased in gastric cancer cells transfected with si-PTEN only, compared with the cells transfected with si-PTEN and treated with MLN8237. These results confirmed 
A
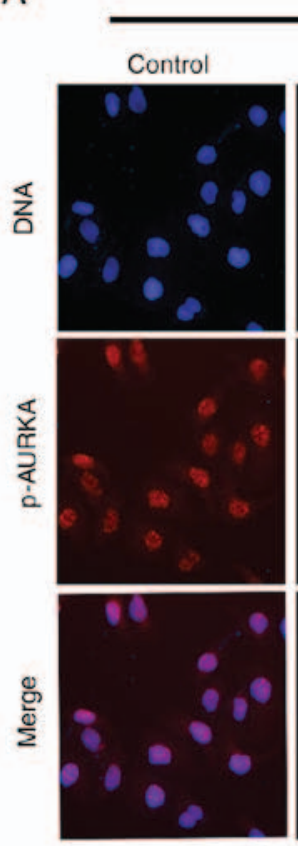

B
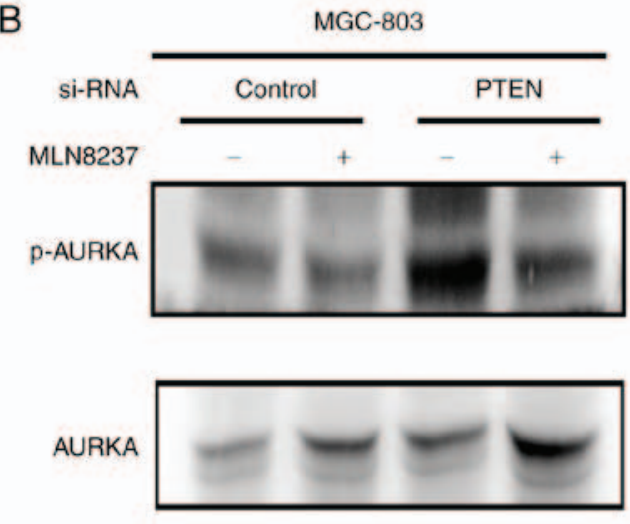

GAPDH

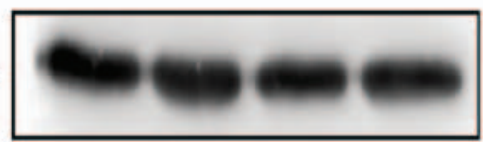

MGC-803

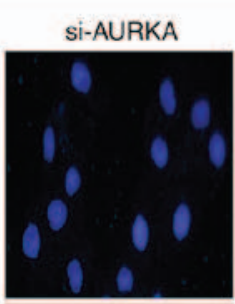

a
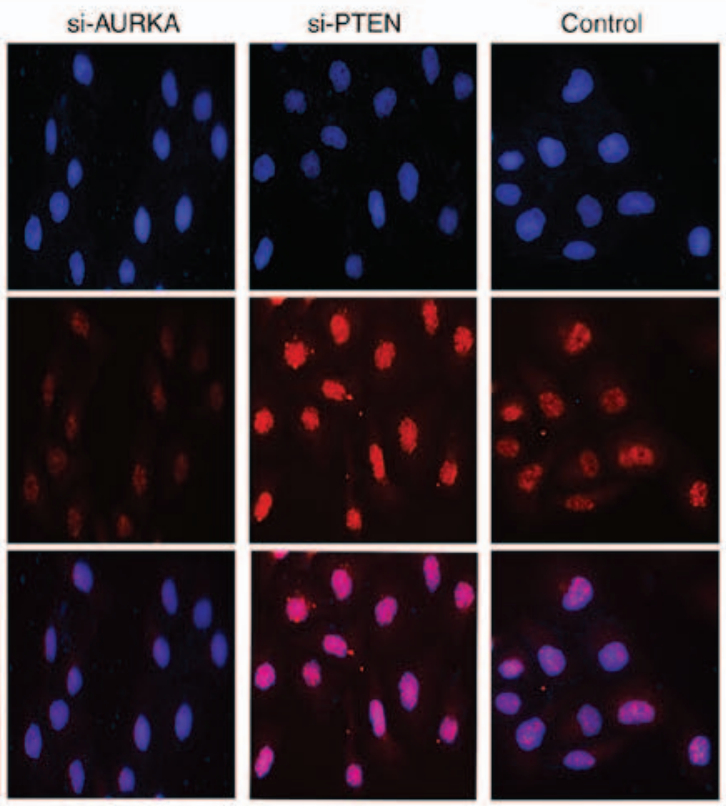

C
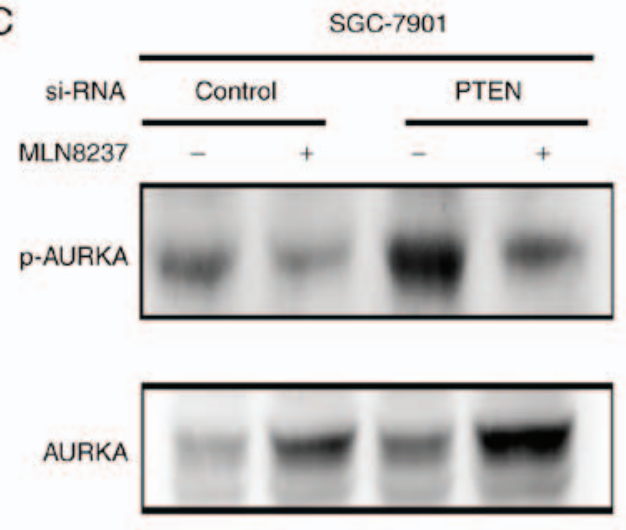

GAPDH

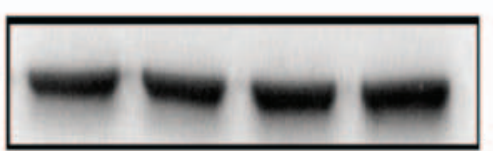

Figure 5. PTEN regulates AURKA by activating p-AURKA. (A) Immunofluorescence analysis of p-AURKA levels showed a significant increase in MGC803 and SGC7901 cells transfected with si-PTEN (magnification, x400). Protein expression levels of AURKA and p-AURKA in (B) MGC803 and (C) SGC7901

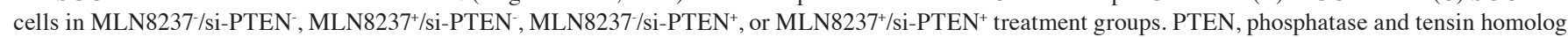
deleted on chromosome 10; AURKA, aurora kinase A; si-, small interfering RNA; p-, phosphorylated.

that the upregulation of p-AURKA by the downregulation of PTEN resulted in the overexpression of AURKA.

Suppression of PTEN affects several signal pathways involved in the development of gastric cancer. According to the present study, PTEN inhibited tumorigenesis by interacting with genes or modulating multiple signal transduction pathways (34-36). To further examine the effects of the reduced expression of PTEN on other intracellular signaling pathways in gastric cancer cells, the expression of key molecules in the signaling pathways of MGC803 and SGC7901 cells were investigated. The MGC803 and SGC7901 cells were transfected with si-PTEN or a control DNA vector, and then analyzed for protein levels of p-AKT, p-GSK3 $\beta$, AKT, GSK $3 \beta$ and $\beta$-catenin. The knockdown of PTEN by si-PTEN transfection resulted in increases in the expression of p-AKT and p-GSK3 $\beta$, compared with that in the control cells, whereas the levels of AKT and GSK $\beta$ did not alter markedly (Fig. 6A). Furthermore, gastric cancer cells showed increased total protein expression levels of $\beta$-catenin and $p$ - $\beta$-catenin (Ser675) following transfection with si-PTEN (Fig. 6B). The cellular distributions of $\beta$-catenin and $\mathrm{p}$ - $\beta$-catenin (Ser675) were also detected in the gastric cancer cells with downregulated PTEN. The downregulation of PTEN led to increases in the protein levels of $\beta$-catenin and $\mathrm{p}-\beta$-catenin (Ser675) in the nucleus and cytoplasm of gastric cancer cells (Fig. 6C and D). The immunofluorescence results also revealed that the expression levels of $\beta$-catenin and $\mathrm{p}$ - $\beta$-catenin (Ser675) in the nucleus and cytoplasm were increased following PTEN knockdown (Fig. 6E and F). These results showed that the knockdown of PTEN resulted in 


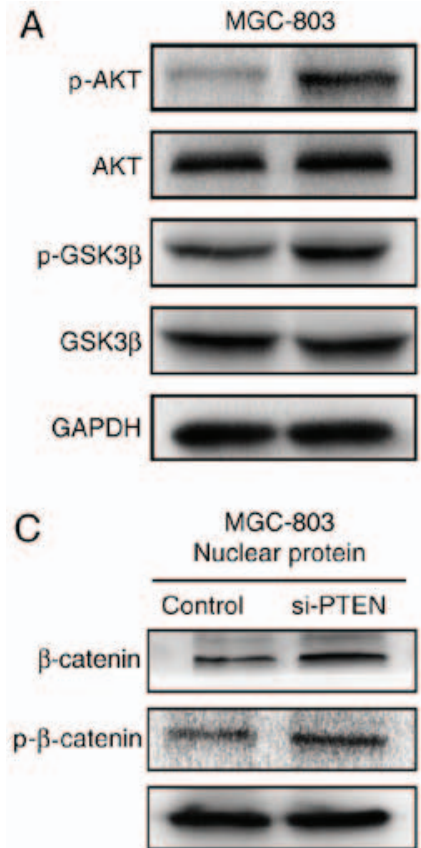

$\mathrm{H3}$

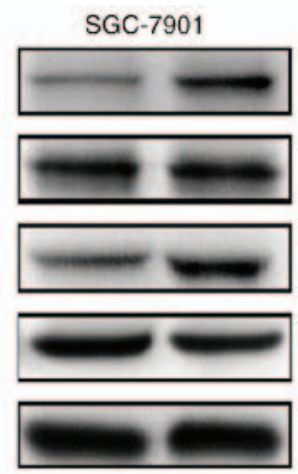

MGC-803 Cytoplasmic protein

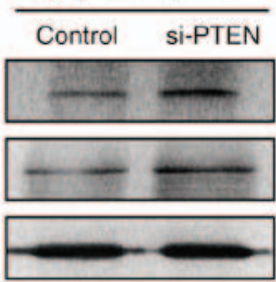

GAPDH

E

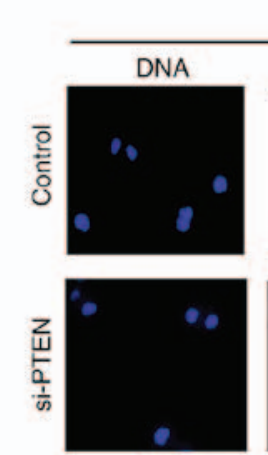

DNA
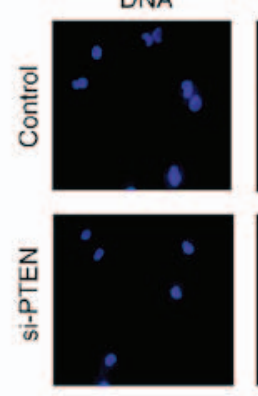

MGC-803
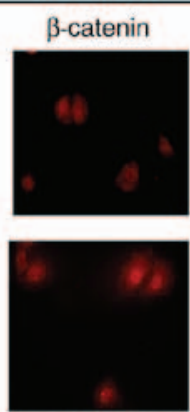

p-ß-catenin
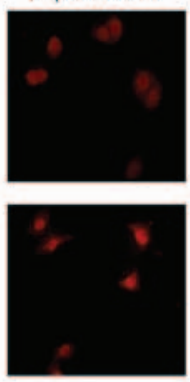
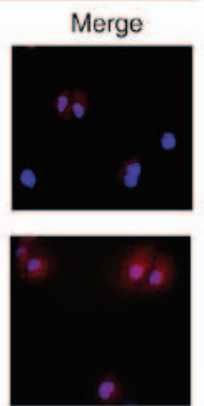

Merge
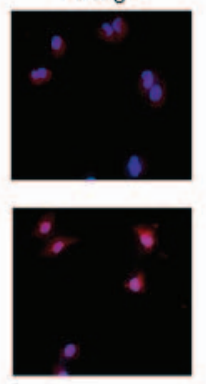
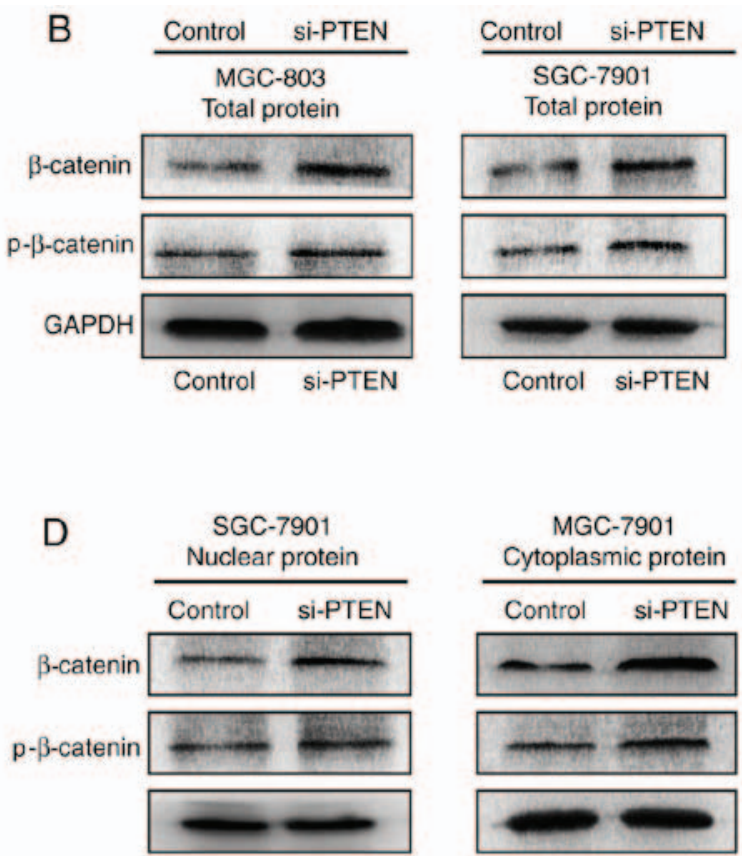

$\mathrm{H} 3$

F

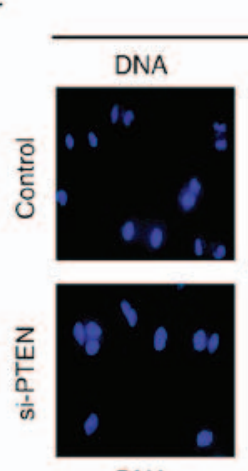

DNA

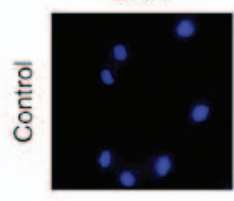

SGC-7901

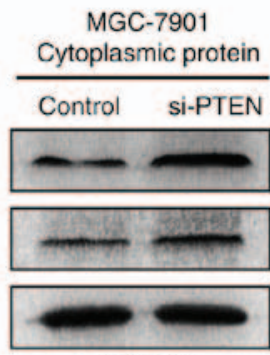

GAPDH

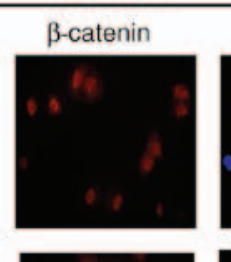

Merge
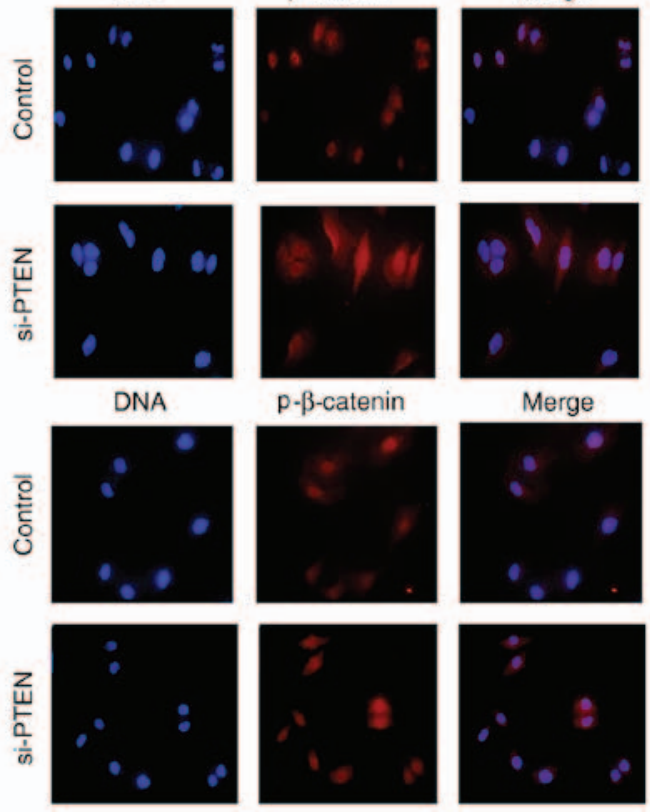

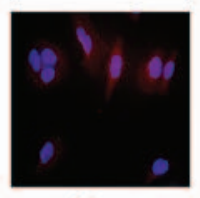

p-ß-catenin
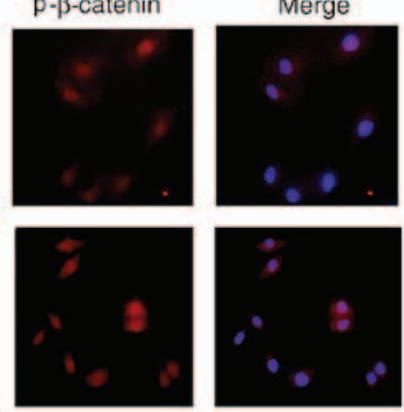

Figure 6. Downregulation of PTEN affects several signaling pathways in gastric cancer. (A) Western blots of protein expression of p-AKT, AKT, p-GSK3 $\beta$ and GSK3 $\beta$ in MGC803 and SGC7901 cells transfected with si-PTEN. (B) Total protein was used to detect protein levels of $\beta$-catenin and p- $\beta$-catenin in MGC803 and SGC7901 cells transfected with si-PTEN. Cytoplasmic and nuclear proteins were extracted, and the levels of $\beta$-catenin and p- $\beta$-catenin were detected in (C) MGC803 and (D) SGC7901 cells transfected with si-PTEN. An immunofluorescence assay was used to detect the levels of $\beta$-catenin and p- $\beta$-catenin and cellular distribution in (E) MGC803 and (F) SGC7901 cells transfected with si-PTEN (magnification, x400). PTEN, phosphatase and tensin homolog deleted on chromosome 10; si-, small interfering RNA; GSK3 $\beta$, glycogen synthase kinase 3 $\beta$; p-, phosphorylated.

increased expression of certain target, intracellular signaling pathway-associated molecules to promote the development of gastric cancer.

\section{Discussion}

Due to the lack of typical symptoms, patients are often diagnosed at an advanced stage of gastric cancer. Although there have been increases in the efficiency of early diagnosis and in the appearance of novel effective chemotherapeutic regimens, the 5-year-survival rate of advanced gastric cancer remains low at $<20 \%$ ), and the majority of patients with advanced-stage or metastatic disease only survive $<1$ year (37). The poor prognosis of patients with gastric cancer indicates that current comprehension of the molecular mechanisms involved in gastric carcinogenesis is lacking. The present study revealed a novel molecular mechanism by which aberrant expression of p-AURKA in gastric cancer cells was crucial 
in the progression of gastric cancer through affecting the PTEN-regulated activity of AURKA. Therefore, p-AURKA represents a drug target warranting further investigation for gastric cancer. In addition, the present study found that knockdown of the expression of PTEN resulted in the upregulation of protein expression levels of p-AKT and p-GSK3 $\beta$, and an increase in protein levels of $\beta$-catenin, which may enhance the carcinogenic properties of gastric cancer cells.

p-AURKA is the product of autophosphorylation of AURKA on the T288 residue in the activation or T-loop, and a number of proteins, including TPX2, NEDD9 and Ajuba, directly associate with AURKA to regulate this process (38). p-AURKA is important in mitosis, phosphorylating BRCA1 protein to reduce $\mathrm{G} 2 / \mathrm{M}$ checkpoint controls (39) and phosphorylating the RAS family protein RALA to regulate mitochondrial fusion, which is critical for the equal post-mitotic segregation of mitochondria between daughter cells (40). AURKA can be autophosphorylated into p-AURKA to promote the progression of gastric cancer. A previous study showed that AURKA directly interacts with important oncogenes and tumor suppressor genes; it phosphorylates Src, stabilizes $\mathrm{N}$-myc, and phosphorylates and downregulates the major tumor suppressor p53 (38). However, the mechanism by which PTEN regulates AURKA remains to be fully elucidated. The present study aimed to examine the regulatory mechanism of PTEN on AURKA. Data were collected from TCGA, and the common PTEN and AURKA gene alterations were analyzed in the cBioPortal for Cancer Genomics, which revealed that PTEN and AURKA had various types of alterations in gastric cancer (Fig. 1). In addition, the expression of PTEN and AURKA were determined in normal gastric mucosa and gastric carcinoma tissues, and their expression in MGC-803, SGC-7901 and GES-I cells were determined at the mRNA and protein levels (Fig. 2). Subsequently, gastric cancer cells were transfected by control vector, si-AURKA and si-PTEN, and it was found that PTEN knockdown using a PTEN siRNA expression vector in MGC-803 and SGC-7901 cells resulted in a significant increase in cell proliferation (Fig. 3A and B). Transwell assays also showed an increase in invasion of si-PTEN-transfected cells and a decrease in invasion of si-AURKA-transfected cells (Fig. 3C). In addition, a high expression of PTEN predicted improved outcomes in patients with gastric cancer, whereas patients with gastric cancer with a high expression of AURKA had poor OS (Fig. 3D and E). Therefore, these results indicated that there is a close association between PTEN and AURKA in carcinogenesis and in the maintenance of malignant phenotypes in gastric cancer cells. In addition, KaplanMeyer analysis indicated that the mRNA expression of PTEN and AURKA may affect the prognosis of stomach adenocarcinoma.

Relational investigations have shown that overexpression of Aurora-A promotes the protein expression of nuclear inhibitor of nuclear factor $(\mathrm{NF})-\kappa \mathrm{B}$ and enhances the activity of $\mathrm{NF}-\kappa \mathrm{B}$, thus promoting the transcription of microRNA-21, which negatively regulates PTEN (41). According to a previous study in mouse embryonic fibroblasts and mouse keratinocyte cell lines, Fbxw7 and PTEN tumor suppressor pathways, which control the levels of the oncoprotein AURKA, and the loss of PTEN attenuates the degradation of Aurora-A by Fbxw7 through the AKT/GSK3 $\beta$ pathway (42). In the present study, the data of 478 patients from TCGA indicated that the expression of PTEN was significantly negatively correlated with the expression of AURKA (Fig. 4A). These results showed that AURKA and PTEN interacted with each other. However, there are no reports on the way in which PTEN affects the expression of AURKA by affecting p-AURKA, which is important for the development of gastric cancer. In the present study, it was observed that AURKA was overexpressed in gastric carcinoma and gastric cancer cell lines at the mRNA and protein levels (Fig. 2). Experiments showed that a low expression of PTEN resulted in a significant decrease in the mRNA and protein expression of AURKA in MGC-803 and SGC-7901 cells (Fig 4C-F). In addition, PTNE knockdown led to elevation in the expression levels of p-AURKA and AURKA in gastric cancer cell lines, whereas AURKA knockdown lead to a reduction in the expression of p-AURKA (Fig. 4E and F). si-PTEN transfection also led to an increase in p-AURKA in the nucleus of MGC803 and SGC7901 cell lines (Fig. 5A). These results suggested that p-AURKA may be one of the downstream targets of PTEN, and the role of p-AURKA in mediating the regulation of AURKA by PTEN requires further clarification. PTEN may downregulate the formation of p-AURKA to affect the function of AURKA. As shown in Fig. 5B and C, PTEN knockdown with MLN8237 treatment led to an increase in the protein levels of AURKA in MGC-803 and SGC-7901 cells, whereas p-AURKA increased more markedly in cells transfected with si-PTEN than in other groups. These results not only further confirmed that p-AURKA is a downstream regulator of PTEN, but also confirmed the close association between PTEN and AURKA.

Based on the above findings, it was confirmed that p-AURKA is important in mediating the PTEN-associated regulation of AURKA, thus indicating its involvement in carcinogenesis and in maintaining the malignant phenotypes of gastric cancer cells. When p-AURKA was inhibited in MGC-803 and SGC-7901 cells by transient transfection with si-AURKA, the protein level of PTEN increased and malignant phenotypes, including increased cell proliferation and invasion, were observed in the gastric cancer cells. Transfection of the gastric cancer cells with si-PTEN led to the increased expression levels of AURKA and p-AURKA, and the overexpression of AURKA has been shown result in the chemoresistance of several malignant tumor cells (43-45) with poorer patient outcomes. These results suggested that p-AURKA induced the malignant phenotype of tumor cells, which revealed that p-AURKA may be a novel target of antitumor drugs. In addition, it was found that the knockdown of PTEN affected several signaling pathways to promote the development of gastric cancer.

Although the incidence and mortality rates of gastric cancer have been in gradual decline globally over the last 30 years, gastric cancer remains a major threat to the health of patients across the world. In previous years, several methods have emerged for the treatment of gastric cancer, including radical surgery, radiotherapy and chemoradiotherapy, and novel technologies have emerged, including laparoscopic gastrectomy (46) and robotic gastrectomy (47). Unlike the favorable prognosis of early gastric cancer, a substantial number patients with advanced gastric cancer experience tumor recurrence during their lifetime, even following radical surgery. Therefore, 
additional strategies are required to improve the survival rate of patients with advanced gastric cancer. However, numerous trials have failed to show an added benefit of chemotherapy to surgery (48). Combination chemotherapeutic regimens produce higher response rates and longer survival rates, compared with single agents, however, therapeutic options remain limited, and the overall prognosis remains poor (5). Based on these findings, as patients with gastric cancer have a high mortality rate, novel therapeutic strategies, useful biomarkers and personalized treatments are required to improve the outcomes of patients with gastric cancer. Therefore, patients with gastric cancer require DNA testing to screen out those with a high expression of AURKA. According to the present study, PTEN downregulated the expression of p-AURKA and further affected the activation of AURKA, and p-AURKA induced the malignant phenotype of tumor cells. A combination of PTEN- and AURKA-targeted therapies may be used for patients who benefit from specific targeted treatments and improve the survival rate of patients. Although several candidate biomarkers and therapeutic targets have been reported, few are used in clinical practice. Therefore, in the future, improvements in next-generation sequencing technology are required to improve the therapy and prognosis of patients with gastric cancer.

In conclusion, the present study presented the novel finding that PTEN inhibited the expression of AURKA by suppressing the activity of p-AURKA and thus regulated the malignant phenotype of gastric cancer cells. These results provide a novel approach, in which the combined targeting of PTEN and AURKA may potentially serve as a therapeutic treatment for gastric cancer, which may enable more accurate gastric cancer cell death that spares normal cells.

\section{Acknowledgements}

The authors would like to express their gratitude to everyone in the Laboratory of Neuro-Oncology, Tianjin Neurological Institute (Tianjin, China) for their technical assistance.

\section{Funding}

This study was supported by grants from the National High Technology Research and Development Program 863 (grant nos. 2014AA021102 and 2016YFC0902502) and from the China National Natural Scientific Fund (grant nos. 81372703 and 81172356).

\section{Availability of data and materials}

The datasets generated and analyzed during the current study are available in the cBioPortal for Cancer Genomics and the KM Plotter online repositories, (http://cbioportal.org, and http://www.kmplot.com).

\section{Authors' contributions}

QL, XL, RW contributed to the conception of the study. YS contributed significantly to analysis and manuscript preparation. LL performed the data analyses and wrote the manuscript. CK and QZ helped perform the analysis with constructive discussions.

\section{Ethics approval and consent to participate}

The present study was approved by the Ethics Committee of Tianjin Medical University General Hospital (Tianjin, China). All subjects provided written informed consent prior to enrollment.

\section{Consent for publication}

All patients whose tissue samples were used gave written informed consent for publication of this study.

\section{Competing interests}

The authors declare that they have no competing interests.

\section{References}

1. Global Burden of Disease Cancer Collaboration, Fitzmaurice C, Allen C, Barber RM, Barregard L, Bhutta ZA, Brenner H, Dicker DJ, Chimed-Orchir O, Dandona R, Dandona L, et al: Global, regional, and national cancer incidence, mortality, years of life lost, years lived with disability, and disability-adjusted life-years for 32 cancer groups, 1990 to 2015: A systematic analysis for the global burden of disease study. JAMA Oncol 3: 524-548, 2017.

2. SEER Cancer Statistics Factsheets: Stomach Cancer. National Cancer Institute, 2016.

3. Arrington AK, Nelson R, Patel SS, Luu C, Ko M, Garcia-Aguilar J and Kim J: Timing of chemotherapy and survival in patients with resectable gastric adenocarcinoma. World J Gastrointest Surg 5: 321-328, 2013

4. Sakar B, Karagol H, Gumus M, Basaran M, Kaytan E, Argon A, Ustuner Z, Bavbek SE, Bugra D and Aykan FN: Timing of death from tumor recurrence after curative gastrectomy for gastric cancer. Am J Clin Oncol 27: 205-209, 2004.

5. Wagner AD, Unverzagt S, Grothe W, Kleber G, Grothey A, Haerting J and Fleig WE: Chemotherapy for advanced gastric cancer. Cochrane Database Syst Rev: Mar 17, 2010 (Epub ahead of print). doi: 10.1002/14651858.CD004064.pub3.

6. Glover DM, Leibowitz MH, McLean DA and Parry H: Mutations in aurora prevent centrosome separation leading to the formation of monopolar spindles. Cell 81: 95-105, 1995.

7. Nguyen AL and Schindler K: Specialize and divide (twice): Functions of three aurora kinase homologs in mammalian oocyte meiotic maturation. Trends Genet 33: 349-363, 2017.

8. Sugimoto K, Urano T, Zushi H, Inoue K, Tasaka H, Tachibana M and Dotsu M: Molecular dynamics of Aurora-A kinase in living mitotic cells simultaneously visualized with histone $\mathrm{H} 3$ and nuclear membrane protein importinalpha. Cell Struct Funct 27: 457-467, 2002.

9. Sen S, Zhou H, Zhang RD, Yoon DS, Vakar-Lopez F, Ito S, Jiang F, Johnston D, Grossman HB, Ruifrok AC, et al: Amplification/overexpression of a mitotic kinase gene in human bladder cancer. J Natl Cancer Inst 94: 1320-1329, 2002.

10. Staff S, Isola J, Jumppanen M and Tanner M: Aurora-A gene is frequently amplified in basal-like breast cancer. Oncol Rep 23: 307-312, 2010.

11. Yang SB, Zhou XB, Zhu HX, Quan LP, Bai JF, He J, Gao YN, Cheng SJ and Xu NZ: Amplification and overexpression of Aurora-A in esophageal squamous cell carcinoma. Oncol Rep 17: 1083-1088, 2007.

12. Kamada K, Yamada Y, Hirao T, Fujimoto H, Takahama Y, Ueno M, Takayama T, Naito A, Hirao S and Nakajima Y: Amplification/overexpression of Aurora-A in human gastric carcinoma: Potential role in differentiated type gastric carcinogenesis. Oncol Rep 12: 593-599, 2004.

13. Maehama T and Dixon JE: PTEN: A tumour suppressor that functions as a phospholipid phosphatase. Trends Cell Biol 9: 125-128, 1999.

14. Ortega-Molina A and Serrano M: PTEN in cancer, metabolism, and aging. Trends Endocrinol Metab 24: 184-189, 2013.

15. Maehama Tand Dixon JE: The tumor suppressor,PTEN/MMAC1, dephosphorylates the lipid second messenger, phosphatidylinositol 3,4,5-trisphosphate. J Biol Chem 273: 13375-13378, 1998. 
16. Manning BD and Cantley LC: AKT/PKB signaling: Navigating downstream. Cell 129: 1261-1274, 2007.

17. VanderLaan PA, Rangachari D, Mockus SM, Spotlow V, Reddi HV, Malcolm J, Huberman MS, Joseph LJ, Kobayashi SS and Costa DB: Mutations in TP53, PIK3CA, PTEN and other genes in EGFR mutated lung cancers: Correlation with clinical outcomes. Lung Cancer 106: 17-21, 2017.

18. Smith IN and Briggs JM: Structural mutation analysis of PTEN and its genotype-phenotype correlations in endometriosis and cancer. Proteins 84: 1625-1643, 2016.

19. Liu JC, Wang DY, Egan SE and Zacksenhaus E: Common and distinct features of mammary tumors driven by Pten-deletion or activating Pik3ca mutation. Oncotarget 7: 9060-9068, 2016.

20. Mina S, Bohn BA, Simon R, Krohn A, Reeh M, Arnold D, Bokemeyer C, Sauter G, Izbicki JR, Marx A and Stahl PR: PTEN deletion is rare but often homogeneous in gastric cancer. J Clin Pathol 65: 693-698, 2012.

21. Livak KJ and Schmittgen TD: Analysis of relative gene expression data using real-time quantitative PCR and the 2(-Delta Delta) C(T) method. Methods 25: 402-408, 2001.

22. Wang J, Nikhil K, Viccaro K, Chang L, White J and Shah K Phosphorylation-dependent regulation of ALDH1A1 by aurora kinase a: Insights on their synergistic relationship in pancreatic cancer. BMC Biol 15: 10, 2017.

23. Pitts TM, Bradshaw-Pierce EL, Bagby SM, Hyatt SL, Selby HM, Spreafico A, Tentler JJ, McPhillips K, Klauck PJ, Capasso A, et al: Antitumor activity of the aurora a selective kinase inhibitor alisertib, against preclinical models of colorectal cancer. Oncotarget 7: 50290-50301, 2016.

24. Chen C, Song G, Xiang J, Zhang H, Zhao S and Zhan Y: AURKA promotes cancer metastasis by regulating epithelial-mesenchymal transition and cancer stem cell properties in hepatocellular carcinoma. Biochem Bioph Res Commun 486: 514-520, 2017.

25. Katsha A, Arras J, Soutto M, Belkhiri A and El-Rifai W: AURKA regulates JAK2-STAT3 activity in human gastric and esophageal cancers. Mol Oncol 8: 1419-1428, 2014.

26. You D, Xin J, Volk A, Wei W, Schmidt R, Scurti G, Nand S, Breuer EK, Kuo PC, Breslin P, et al: FAK mediates a compensatory survival signal parallel to PI3K-AKT in PTEN-null T-ALL Cells. Cell Rep 10: 2055-2068, 2015.

27. Yang LY, He CY, Chen XH, Su LP, Liu BY and Zhang H: Aurora kinase a revives dormant laryngeal squamous cell carcinoma cells via FAK/PI3K/Akt pathway activation. Oncotarget 7 : 48346-4859, 2016.

28. Wang Y, Chen B, Wang Z, Zhang W, Hao K, Chen Y, Li K, Wang T, Xie Y, Huang Z and Tong X: Marsdenia tenacissimae extraction (MTE) inhibits the proliferation and induces the apoptosis of human acute $\mathrm{T}$ cell leukemia cells through inactivating $\mathrm{PI} 3 \mathrm{~K} / \mathrm{AKT} / \mathrm{mTOR}$ signaling pathway via PTEN enhancement. Oncotarget 7: 82851-82863, 2016.

29. Li JP, Yang YX, Liu QL, Pan ST, He ZX, Zhang X, Yang T, Chen XW, Wang D, Qiu JX and Zhou SF: The investigational aurora kinase a inhibitor alisertib (MLN8237) induces cell cycle G2/M arrest, apoptosis, and autophagy via p38 MAPK and Akt/mTOR signaling pathways in human breast cancer cells. Drug Des Devel Ther 9: 1627-1652, 2015.

30. Jiang $X$ and Li H: Overexpression of LRIG1 regulates PTEN via MAPK/MEK signaling pathway in esophageal squamous cell carcinoma. Exp Ther Med 12: 2045-2052, 2016.

31. Puig-Butille JA, Vinyals A, Ferreres JR, Aguilera P, Cabré E, Tell-Martí G, Marcoval J, Mateo F, Palomero L, Badenas C, et al: AURKA overexpression is driven by FOXM1 and MAPK/ERK activation in melanoma cells harboring BRAF or NRAS mutations: Impact on melanoma prognosis and therapy. J Invest Dermatol 137: 1297-1310, 2017.
32. Zorba A, Buosi V, Kutter S, Kern N, Pontiggia F, Cho YJ and Kern D: Molecular mechanism of aurora a kinase autophosphorylation and its allosteric activation by TPX2. Elife 3: e02667, 2014.

33. Shu LP, Zhou ZW, Zi D, He ZX and Zhou SF: A SILAC-based proteomics elicits the molecular interactome of alisertib (MLN8237) in human erythroleukemia K562 cells. Am J Trans Res 7: 2442-2461, 2015

34. Narbonne P, Maddox PS and Labbe JC: DAF-18/PTEN signals through AAK-1/AMPK to inhibit MPK-1/MAPK in feedback control of germline stem cell proliferation. PLoS Genet 13: e1006738, 2017.

35. Benitez JA, Ma J, D'Antonio M, Boyer A, Camargo MF, Zanca C, Kelly S, Khodadadi-Jamayran A, Jameson NM, Andersen M, et al: PTEN regulates glioblastoma oncogenesis through chromatin-associated complexes of DAXX and histone H3.3. Nat Commun 8: 15223, 2017.

36. de la Rosa J, Weber J, Friedrich MJ, Li Y, Rad L, Ponstingl H, Liang Q, de Quirós SB, Noorani I, Metzakopian E, et al: A single-copy sleeping beauty transposon mutagenesis screen identifies new PTEN-cooperating tumor suppressor genes. Nat Genet 49: 730-741, 2017.

37. Shah MA: Gastrointestinal cancer targeted therapies in gastric cancer-the dawn of a new era. Nat Rev Clin Oncol 11: 10-11,2014.

38. Shagisultanova E, Dunbrack RL Jr and Golemis EA: Issues in interpreting the in vivo activity of Aurora-A. Expert Opin Ther Targets 19: 187-200, 2015.

39. Ouchi M, Fujiuchi N, Sasai K, Katayama H, Minamishima YA, Ongusaha PP, Deng C, Sen S, Lee SW, Ouchi T: BRCA1 phosphorylation by Aurora-A in the regulation of G2 to M transition. J Biol Chem 279: 19643-19648, 2004.

40. Kashatus DF, Lim KH, Brady DC, Pershing NL, Cox AD and Counter CM: RALA and RALBP1 regulate mitochondrial fission at mitosis. Nat Cell Biol 13: 1108-1115, 2011.

41. Zhang K, Chen J, Chen D, Huang J, Feng B, Han S, Chen Y, Song H, De W,Zhu Z, et al: Aurora-A promotes chemoresistance in hepatocelluar carcinoma by targeting NF-kappaB/microRNA-21/PTEN signaling pathway. Oncotarget 5: 12916-12935, 2014.

42. Kwon YW, Kim IJ, Wu D, Lu J, Stock WA Jr, Liu Y, Huang Y, Kang HC, DelRosario R, Jen KY, et al: Pten regulates Aurora-A and cooperates with Fbxw7 in modulating radiation-induced tumor development. Mol Cancer Res 10: 834-844, 2012.

43. Cirak Y, Furuncuoglu Y, Yapicier O, Aksu A and Cubukcu E: Aurora A overexpression in breast cancer patients induces taxane resistance and results in worse prognosis. J BUON 20: 1414-1419, 2015.

44. Mignogna C, Staropoli N, Botta C, De Marco C, Rizzuto A Morelli M, Di Cello A, Franco R, Camastra C, Presta I, et al: Aurora Kinase A expression predicts platinum-resistance and adverse outcome in high-grade serous ovarian carcinoma patients. J Ovarian Res 9: 31, 2016.

45. Xu J, Yue CF, Zhou WH, Qian YM, Zhang Y, Wang SW, Liu AW and Liu Q: Aurora-A contributes to cisplatin resistance and lymphatic metastasis in non-small cell lung cancer and predicts poor prognosis. J Transl Med 12: 200, 2014.

46. Kitano S, Shiraishi N, Uyama I, Sugihara K, Tanigawa N and Japanese Laparoscopic Surgery Study G: A multicenter study on oncologic outcome of laparoscopic gastrectomy for early cancer in Japan. Ann Surg 245: 68-72, 2007.

47. Yang SY, Roh KH, Kim YN, Cho M, Lim SH, Son T, Hyung WJ and Kim HI: Surgical outcomes after open, laparoscopic, and robotic gastrectomy for gastric cancer. Ann Surg Oncol 24: 1770-1777, 2017.

48. Choi YY, Noh SH and Cheong JH: Evolution of gastric cancer treatment: From the golden age of surgery to an era of precision medicine. Yonsei Med J 56: 1177-1185, 2015. 